\title{
Design and Control of Underwater Robots with Rotating Thrusters
}

\author{
Ali Jebelli*, M. C.E. Yagoub*, B. S. Dhillon** \\ * School of Electrical Engineering and Computer Science, University of Ottawa \\ ** Department of Mechanical Engineering, University of Ottawa
}

\section{Article Info \\ Article history: \\ Received Aug 15, 2016 \\ Revised Oct 28, 2016 \\ Accepted Nov 10, 2016}

\section{Keyword:}

Autonomous underwater vehicle Energy consumption

Mobile thruster

Mass shifter

\begin{abstract}
Among other robots, underwater robot design involves critical control issues due to complex non-linear force and turns controlling. In this paper, a robust approach was proposed to efficiently control the behavior of an underwater robot through five degrees of freedom. Also, by designing a new type of a pair of thruster with the ability to 360 degree rotation along with a mass shifter, it gives this possibility to the robot that easily and with a minimal energy, change its depth quickly, preserving its balance best possible at the same time.
\end{abstract}

\section{Corresponding Author:}

Ali Jebelli,

School of Electrical Engineering and Computer Science,

University of Ottawa,

800 King Edward, Ottawa, Ontario, Canada, K1N 6N5,

Email: ajebelli@uottawa.ca

\section{INTRODUCTION}

Autonomous Underwater Vehicles (AUVs) generally use fixed thrusters for their movements. However, such thrusters should be all "on" to create a simultaneous vertical-horizontal movement to reach a desired depth/position; it will then lead to high-energy consumption and negative impact on robot speed and maneuverability [1-3].

In this work, a research robot prototype was designed with two mobile thrusters that can rotate in a specific angle so that both required vertical and horizontal forces are provided simultaneously (Figure 1 and Figure 2).

Furthermore, the variation of the thruster angle has been made possible by the instant movement of an engine stopper, which consumes much less power than the constant movement of a thruster. Also, a mass shifter was included to make possible the maneuver in the direction of the pitch. All these features have made the proposed design innovative and energy-effective. In fact, the proposed prototype can move under two modes. In the first mode, the device should, by the help of the mass shifter, have a constant horizontal movement and its movement towards vertical and horizontal directions should be achieved through a change in the thruster angle. In the second mode, the thrusters should be kept fixed in a horizontal direction and the vertical movement should be made possible through a change in the body angle in the pitch direction. The first mode is used wherever there is little room of maneuver or to contour/pass obstacles while the second mode is used to preserve and store the source of energy and change in great depths. Note that this structure is innovative and highly energy-saving. 


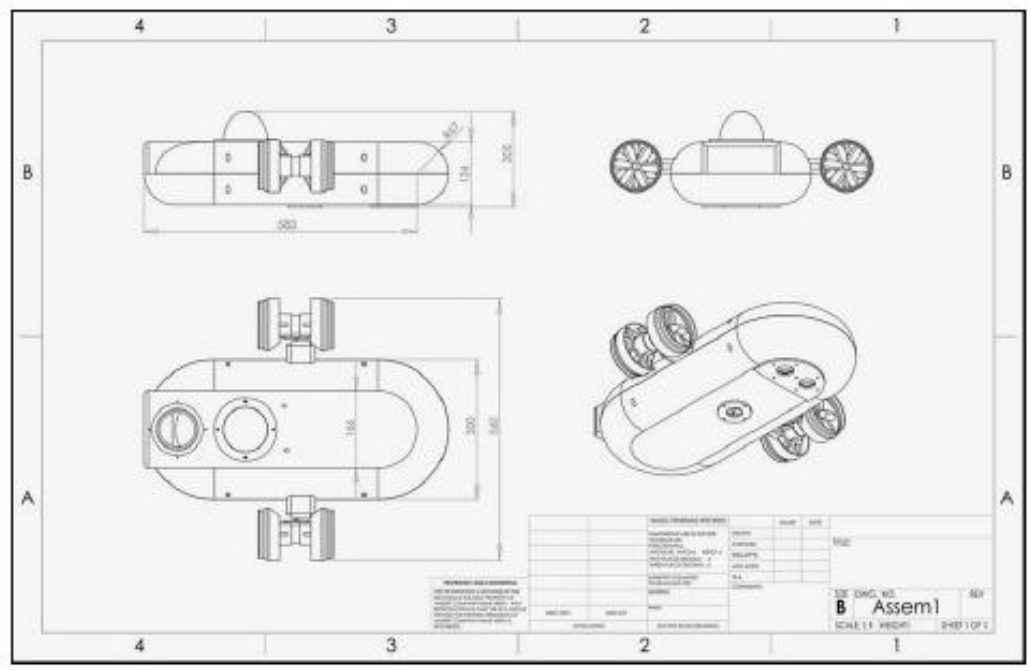

Figure 1. Isometric scheme of the designed robot

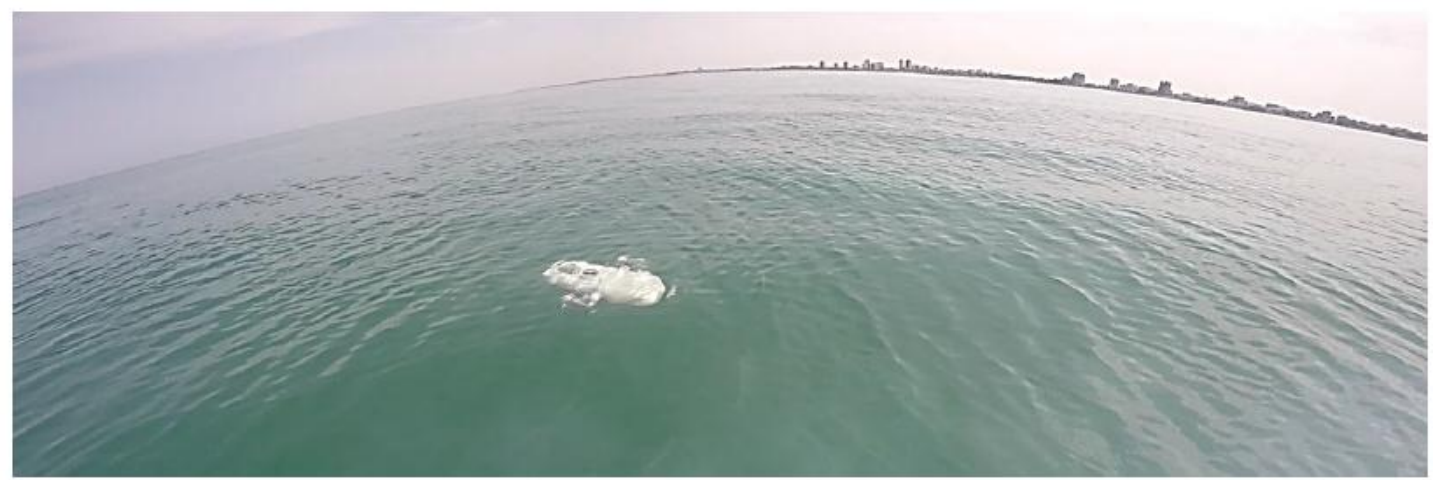

Figure 2. The designed robot in the sea

\section{ROBOT DESIGN}

The robot has five degrees of freedom:

- Horizontal movement

- Vertical movement

- Rotating around X body axis

- Rotating around Y body axis

- Rotating around $\mathrm{Z}$ body axis

To efficiently manage them, five controlling algorithms have been developed and implemented. They involve the following (Figure 3):

- Speed controller (associated with the device horizontal movement)

- Depth controller (Corresponding to the device vertical movement)

- Roll controller

- Pitch controller

- Yaw controller 


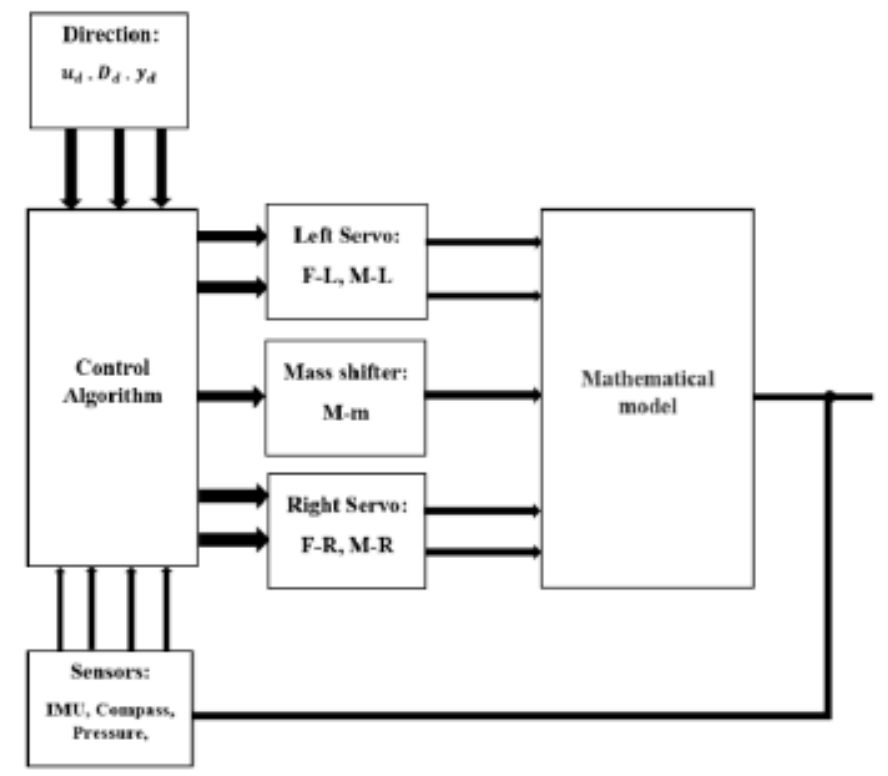

Figure 3. Control Algorithm

\subsection{Speed controller algorithm}

This algorithm sets the speed of the device by adjusting that of the left and right thrusters through a proportional integral controller (PI), which output can be expressed in terms of engine revolution percentage as [4], [5]:

$$
T=k p u \times \varepsilon u+k i u \int \varepsilon u
$$

where $k p u$ is the speed controller proportional coefficient, $k i u$ the speed controller integral coefficient, and $\varepsilon u$ is the speed error defined as:

$$
\varepsilon u=u d-u
$$

where $u d$ and $u$ state for the desired speed and measured speed, respectively.

sub

\subsection{Pitch controller algorithm}

A mass moving system has been used to control the pitch angle. To induce either positive or negative angle towards horizon, a mass moves towards the longitudinal direction of the body (x-axis) causing a change in the center of gravity. So, knowing that the steady state is inherently the zero angle, the mass is moved accordingly to the command (dive or rise). However, by varying the device nose angle relatively to the X-axis, the friction with the fluid will decrease thus, increasing the speed of the robot. A pitch controller was then implemented to adjust the movement of the poise based on the following relation [6]:

$$
S G 2 B B=k p p \times \varepsilon p
$$

where $S_{2} B B$ is the controller output, which includes the poise distance towards the center of balance. $k p_{p}$ is the controller proportional coefficient and $\varepsilon_{\mathrm{p}}$ is the nose angle error calculated as below:

$$
\varepsilon p=\theta d-\theta
$$

with $\theta_{\mathrm{d}}$ the desired pitch angle and $\theta$ the pitch angle measured by sensors. Preliminary simulations demonstrated that a proportional controller is sufficient to reach the desired control (no need for an integrator term).

\subsection{Depth algorithm}

To effectively reach the desired depth, a controller was designed and operated by a depth controller algorithm consisting on two parts. 


\subsubsection{Depth controller at rest}

When the device reaches the desired depth, the depth controller maintain s the desired depth by adjusting the level of engines revolution. For this, a proportional integral controller has been used which output (in \%) can be expressed as [5], [7]:

$$
T=k p D 1 \times \varepsilon D+k i D 1 \int \varepsilon D
$$

where $k p_{D 1}$ is the depth controller proportional coefficient at rest, $k i D_{1}$ the depth controller integral coefficient at rest, and $\varepsilon D$ the depth error, calculated as follows:

$\varepsilon D=D d-D$

with $D d$ the desired depth and $D$ the depth measured by sensors.

\subsubsection{Depth controller during movement}

During the movement, the engine changes the depth angle to provide the required horizontal-vertical forces. For this purpose, a proportional integral controller has been used to control the desired angle such as [5], [7]:

$$
\theta_{s}=k p D 2 \times \varepsilon D+k i D 2 \int \varepsilon D
$$
movement.

with $\mathrm{kpD} 2$ and $k i D_{2}$ the respective depth controller proportional and integral coefficients during

\subsection{Yaw controller}

To maintain the robot in a desired direction, a yaw controller was set by considering the difference in angle between the left and right servos. For example, to rotate towards the right direction, the left servo is placed upper the right servo to become closer to horizon, and inversely, to turn left, the right servo comes up. To implement this logic, a proportional-integral controller has been used which output (in terms of angle difference among servos) can be expressed as [7]:

$$
\theta e=k p y \times \varepsilon y+k i y \int \varepsilon y
$$

where $k p_{y}$ is the proportional coefficient and $k i y$ the integral coefficient of the controller. $\varepsilon y$ is the direction error, calculated as:

$$
\varepsilon y=y d-y
$$

with yd the desired direction angle and y the direction angle determined by the sensors.

\section{SENSORES \\ 3.1. IMU Sensor}

A MPU-9250 Inertial Measurement Unit (IMU) sensor from InvenSense Inc. has been used [8]. It is composed of three sensors, i.e., an acceleration counter to measure the acceleration of the device, its balance and its deviation, a gyroscope to measure the circulation rate of the device and finally, a magnometer to determine the position of the device relatively to the North Pole.

\subsection{Compass Sensor}

A compass sensor has been also incorporated to determine the heading point of the robot. Because the magnometer sensor is highly sensitive to electromagnetic noise and earth magnetic field intensity, its operation in submarine conditions could be not precise. Therefore, we had to integrate a separate digital compass sensor to increase accuracy. The selected compass sensor is a Honeywell HMC6343, a fully integrated electronic compass module that can evaluate the heading direction within a couple of degrees. It is tilt compensated and calibrated to handle magnetic distortions. It combines 3-axis magneto-resistive sensor and 3-axis MEMS accelerometer and compute a heading direction every 200ms [9]. 


\subsection{Pressure Sensor}

Because there is a linear relation between water pressure and water depth, we used a pressure sensor to measure the device depth. For an utmost depth of $20 \mathrm{~m}$, the utmost pressure will be around 3 bar. The selected pressure sensor, the Measurement Spec. MS5803-14BA, is a high resolution pressure sensor with $\mathrm{I}_{2} \mathrm{C}$ interface, optimized for depth measurement systems with a water depth resolution of around $1 \mathrm{~cm}$ [10].

\section{PERFORMANCE RESULTS}

\subsection{Robot speed}

To test the implemented algorithms as well as the associated hardware, we first selected different desired speed values as input commands. As shown in Fig. 4, the robot response follows closely the command after few ms, while the associated left and right engines power in terms of engine revolution percentage are depicted in Figs. 5 and 6, respectively.

Figure 7 shows the body acceleration towards the longitudinal axis of the body. As expected, any increase in the engine revolution implies a similar acceleration, thus speed. However, by rising the speed, the drag force entering into the device from the fluid will act against the thrust, hence decreasing the acceleration to zero and consequently leading to a constant speed.

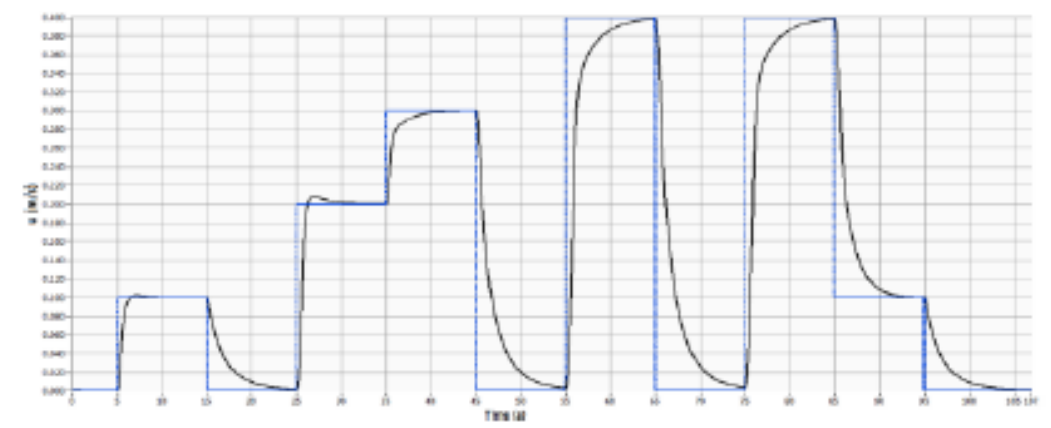

Figure 4. (Blue) desired speed values used as inputs, (Black) robot response

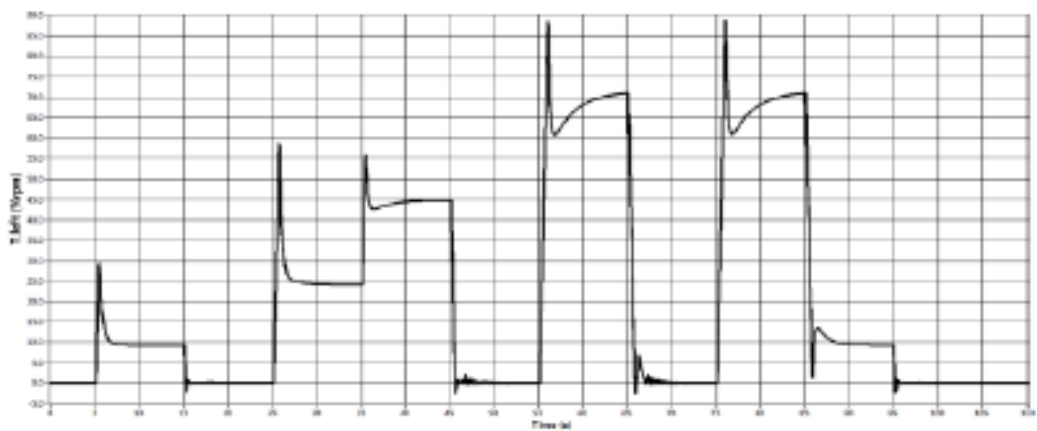

Figure 5. Left engine revolution level

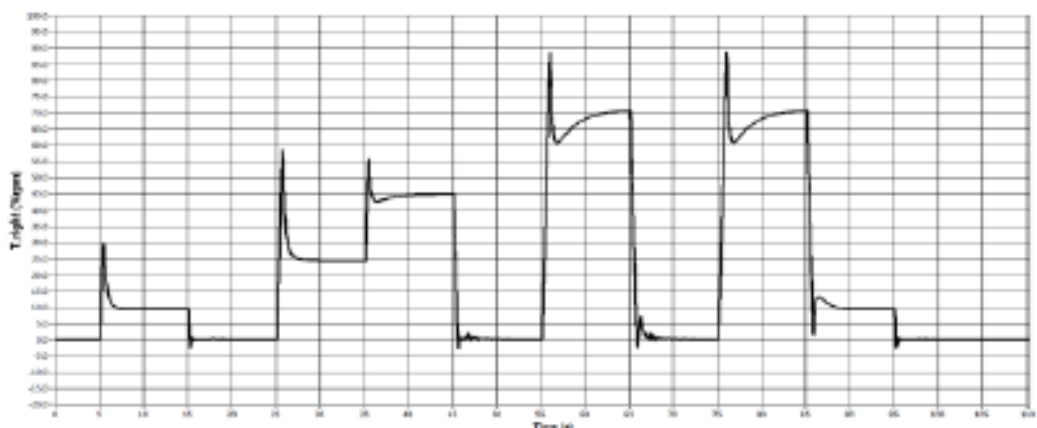


Figure 6. Right engine revolution level

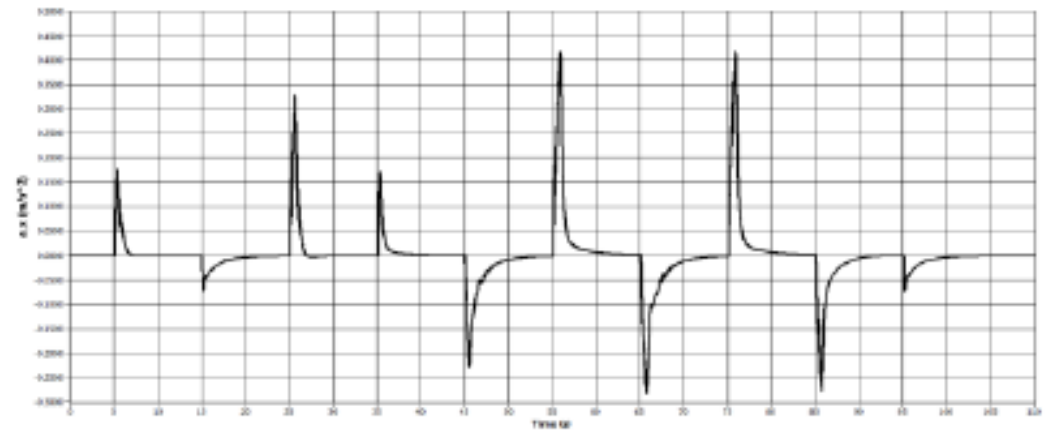

Figure 7. Acceleration towards X-axis.

\subsection{Controller}

To test the robot's designed controller, we selected different depths as shown in figure 8 , leading to the response displayed in figure 9. As shown in Figure 10, the robot response follows quite closely the command in a quite stable way.

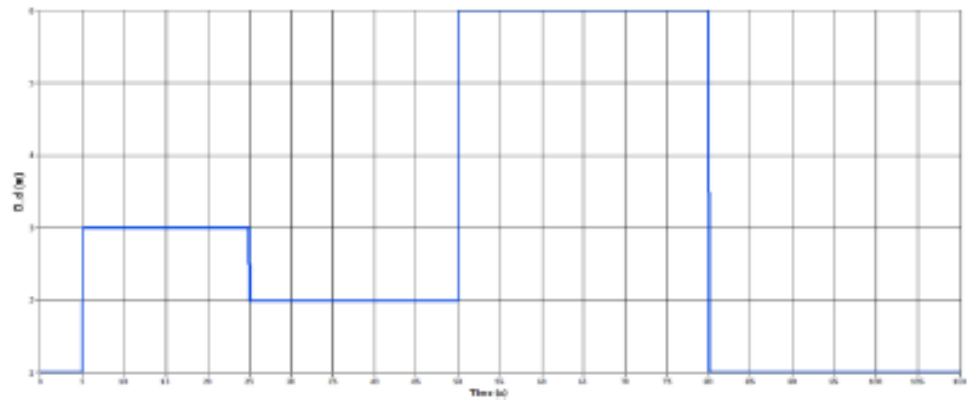

Figure 8. (Blue) Selected depth values

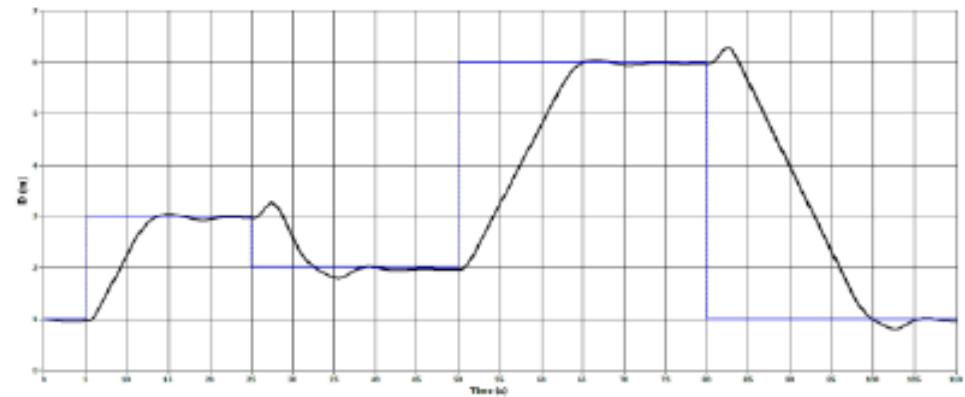

Figure 9. Robot's response to selected depths

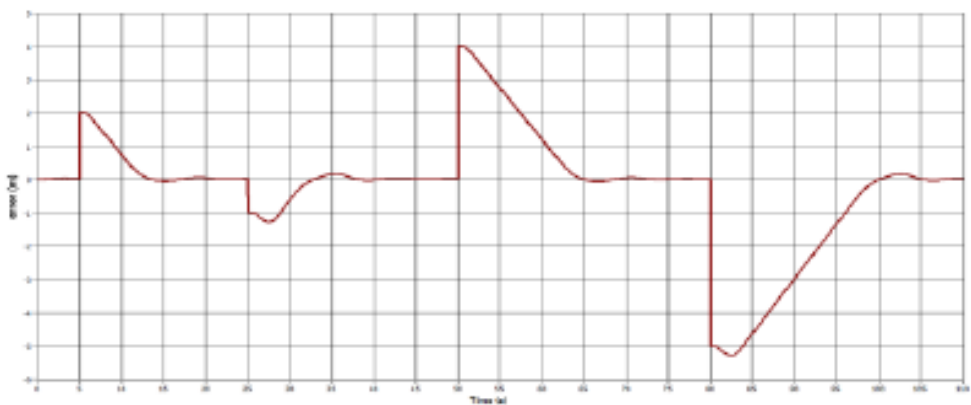

Figure 10. Robot's response: depth balance error 
This can be further demonstrated by focusing on the location of the moving poise during the change of depths, as displayed in figure 11. As noted from this figure, the displacement of the poise followed exactly the depth changes; then, it reached the balance state, i.e., the zero spot. Note that the poise displacement is limited to a maximum of $8 \mathrm{~cm}$ forwards $(+8)$ and $8 \mathrm{~cm}$ backwards $(-8)$.

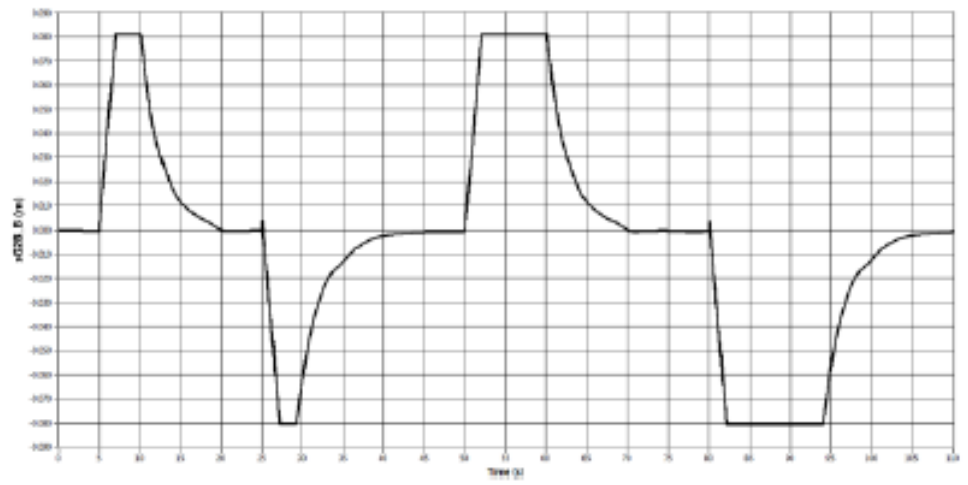

Figure 11. The position of the moving weight (Mass shifter)

Figure 12 shows the nose angle chart for the mentioned input. It can be linked to figure 11 where when the poise has had positive displacement (moving forward), the nose comes down and when the weight has had negative displacement, i.e., moving towards the backside of the balance center, the nose has gone up.

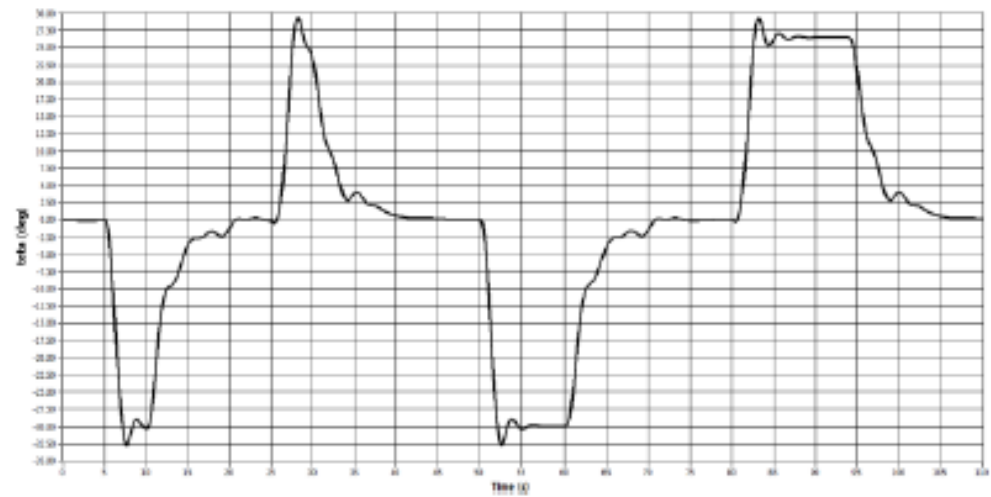

Figure 12. Pitch angle

\subsection{Depth test}

In this test, the robot was programmed for a constant speed of $0.2 \mathrm{~m} / \mathrm{s}$ and a desired targeted depth of $5 \mathrm{~m}$. As shown in figure 13, the depth has been maintained close to the desired value of $5 \mathrm{~m}$. Consequently, the vertical speed of the robot has been kept close to zero (Figure 14). The pitch angle (Figure 15) as well as the poise displacement (Figure 16) closely followed the above commands.

Note that, as displayed in figures 17 and 18, left and right servos have been set in an angle of $-12.5^{\circ}$ to allow the robot reaching the desired depth under fixed operating conditions, i.e., under a speed of $0.2 \mathrm{~m} / \mathrm{s}$.

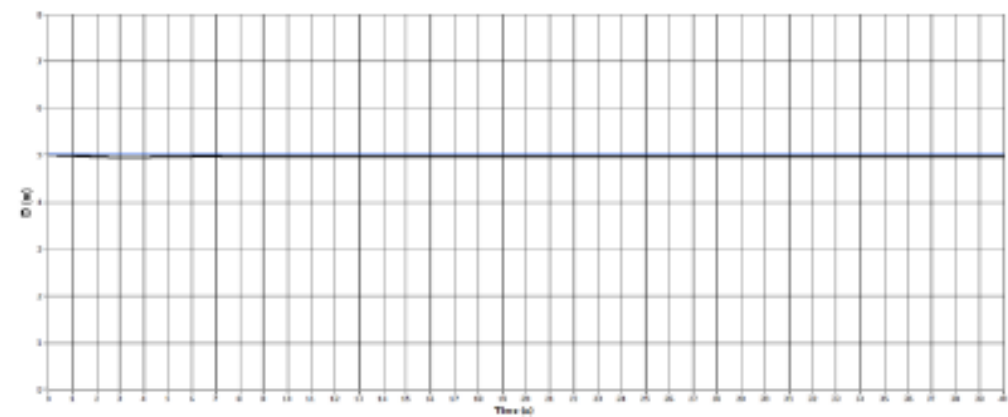

Figure 13. Depth 


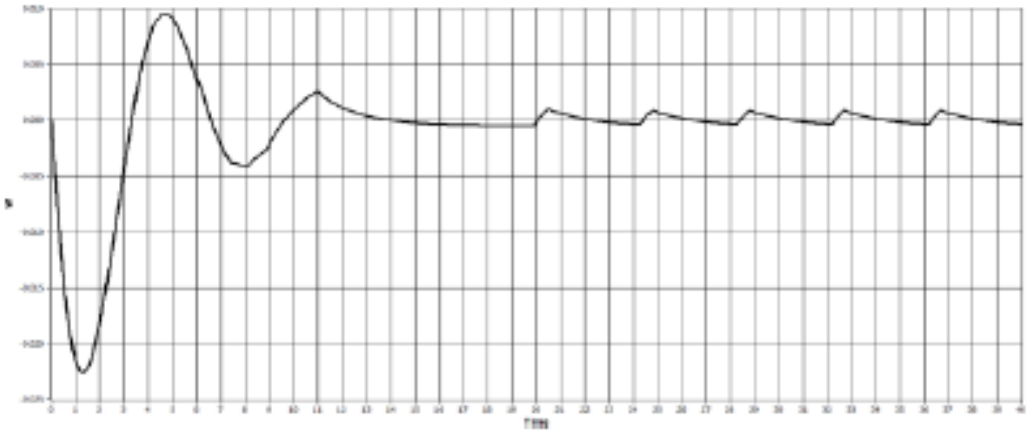

Figure 14. The vertical speed

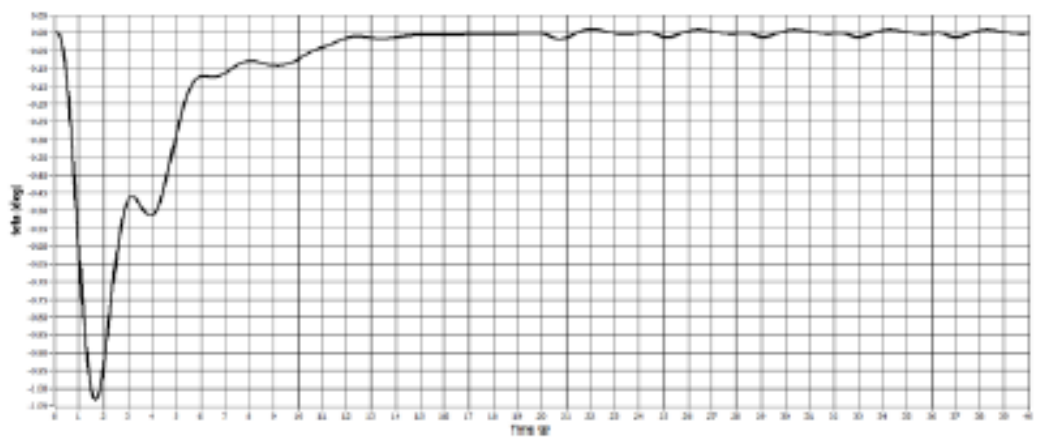

Figure 15. Pitch angle

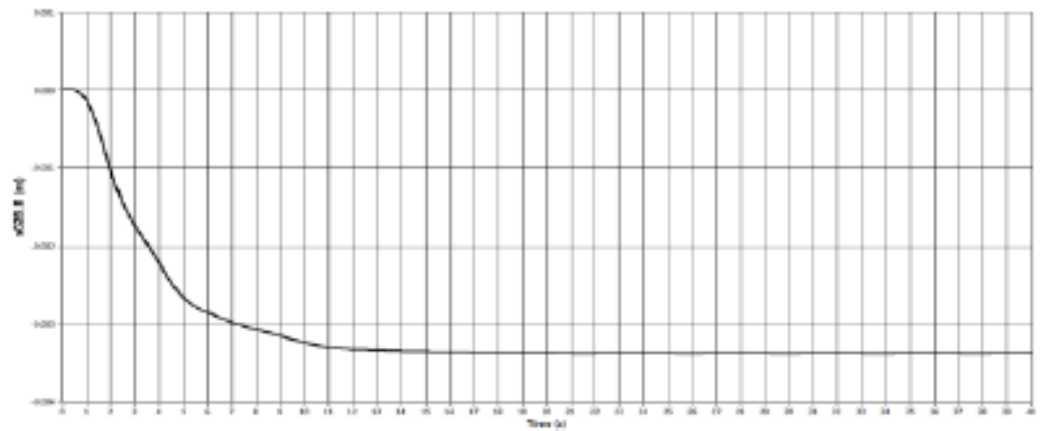

Figure 16. The position of moving weight (Mass shifter)

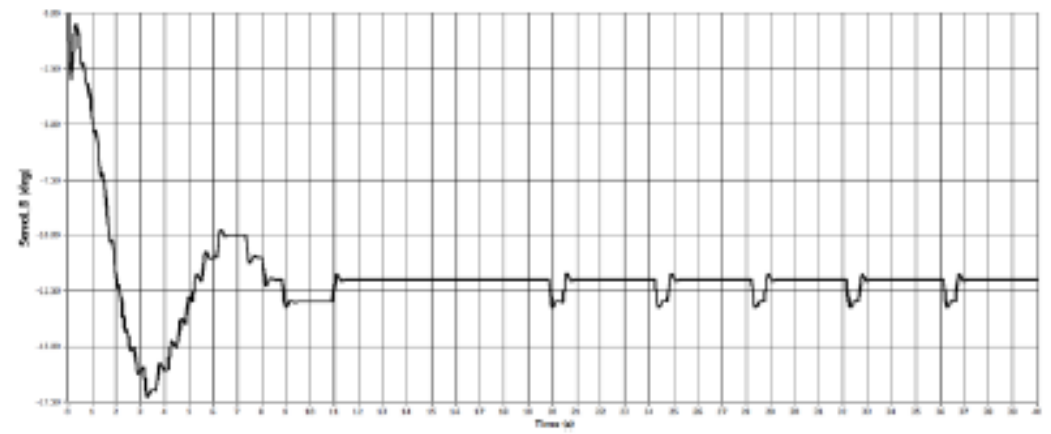

Figure 17. Left servo angle 


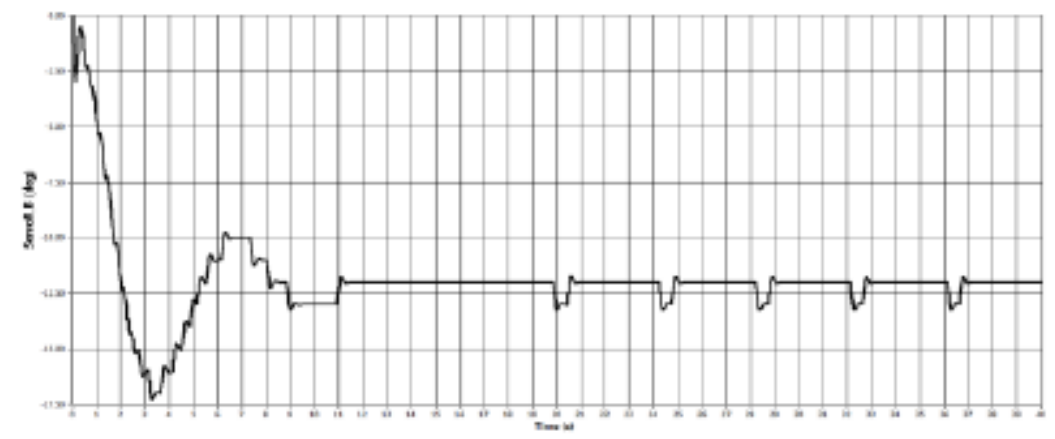

Figure 18. Right servo angle

To further investigate the performance of the designed robot, we set a command close to the limits of the device operation. We set the speed to $0.1 \mathrm{~m} / \mathrm{s}$ while keeping the desirable depth to $5 \mathrm{~m}$. Note that if the device is moving at a slower rate, the angle of servos towards body will increase, becoming close to $-90^{\circ}$. As shown in figures 19 to 23 , the robot reacted well to the commands.

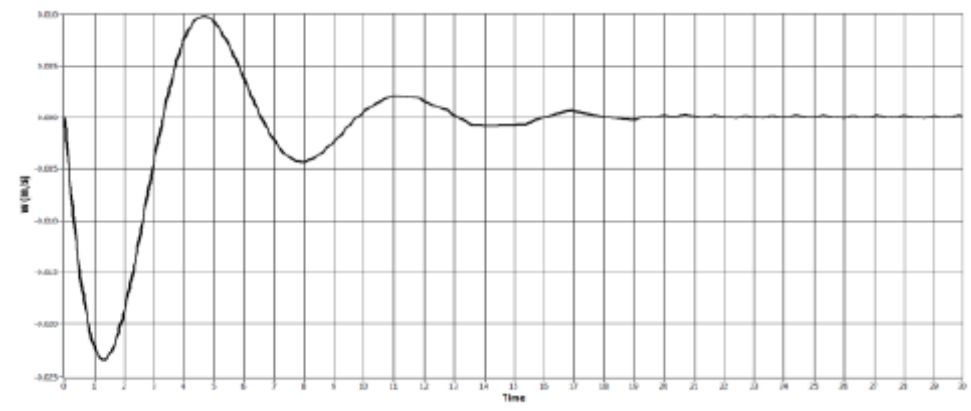

Figure 19. Vertical speed

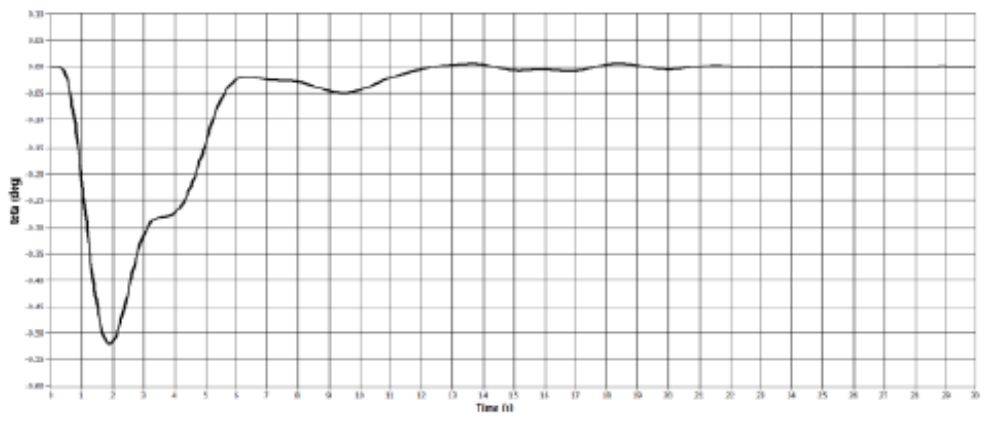

Figure 20. Pitch angle

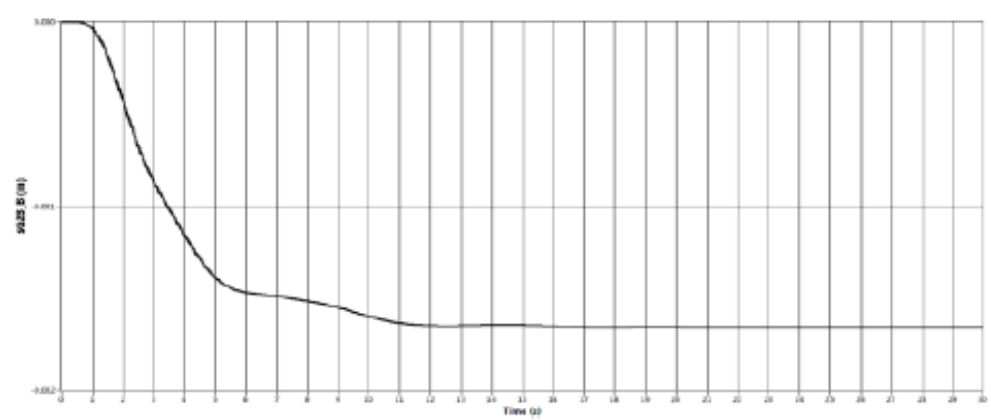

Figure 21. Location of the moving weight (Mass shifter) 


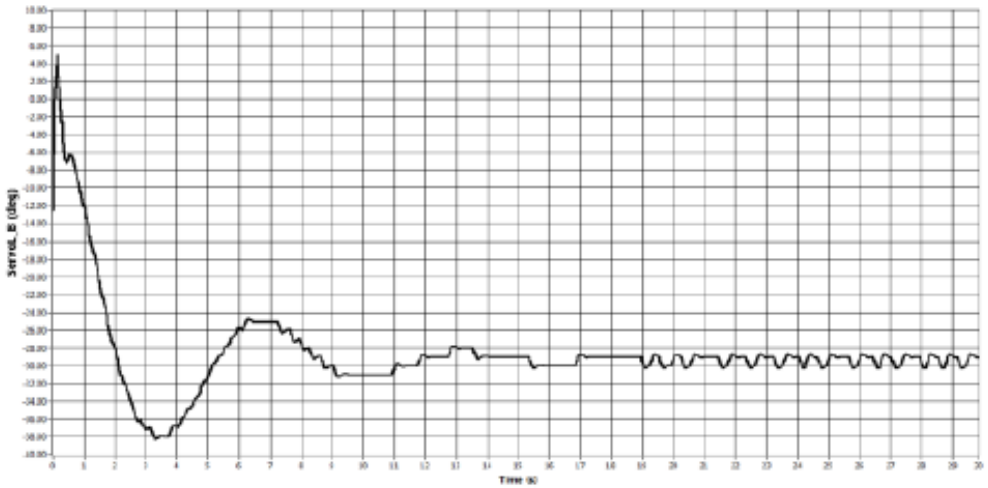

Figure 22. Left servo angle

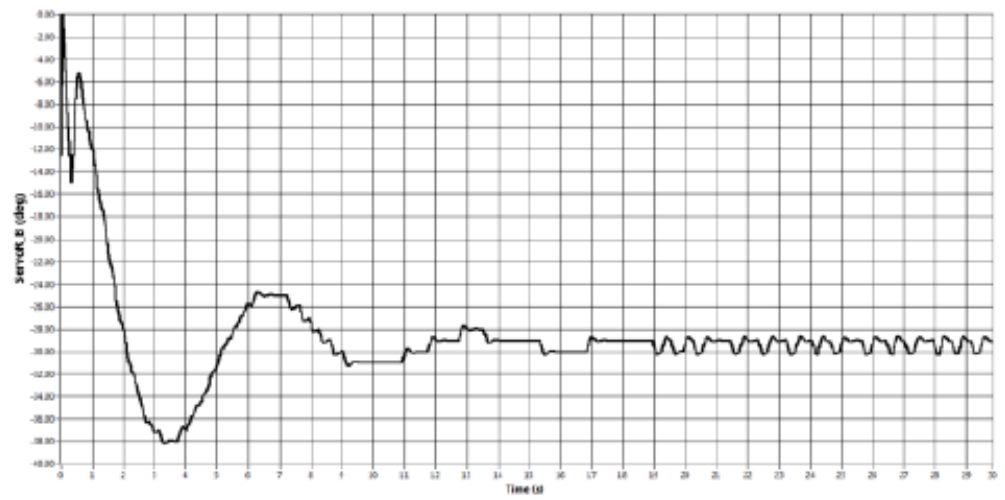

Figure 23. Right servo angle

As anticipated, by slowing down the horizontal speed, the servos' angle increased and then stabilized to $-30^{\circ}$ to reach the defined depth.

\section{REFERENCES}

[1] Virginia TechAutonomous Underwater Vehicle, 2010 Competition TeamMicah Boswell, Craig Tripp, Ben Guzzardi, Joe Ball, Stephen Portner.

[2] V. A. Asare, R. S. Shende. "Mechatronics and Motionability of an Underwater Robot: A Review, " International Journal of Scientific Engineering and Research, Volume 2, Issue3, 2014.

[3] J. YUH." Design and Control of Autonomous Underwater Robots: A Survey,"Autonomous Robots, Volume 8, Issue 1, pp 7-24, Jan 2000.

[4] Kemal Ari, Faik Tekin Asal and Mert Coşgun "Project report on PI, PD, PID controllers "under Middle East Technical University,2012.

[5] Åström, K.J., Control System Design, Lecture notes for ME 155A, Department of Mechanical \& Environmental Engineering, University of California, 2002.

[6] J. Petrich, W.L.Neu and D.J.Stilwell. Identification of a simplied AUV pitch axis model for control design: Theory and experiments. Virginia Polytechnic Institute and State University, 2001.

[7] Vervoort, J. H. A. M., Modeling and Control of Unmanned Underwater Vehicle, Master Thesis, University of Canterbury, 2009.

[8] MPU-9250 Product Specification, InvenSense, 2014

[9] Three-axis Compass with Algorithms HMC6343, Honeywell International Inc, 2014.

[10] DA5803-14BA_006 www.meas-spec. com. March 25, 2013. 000058031641 ECN1973. 


\section{BIOGRAPIES OF AUTHORS}

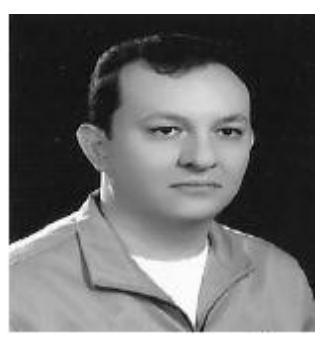

Ali Jebelli received his Bs.c. degree in Electrical Engineering in 2004. He resived his Master of Engineering in Electrical-Mechatronics \& Automatic Control from University Technology Malaysia and Master of Applied Science in Electrical and Computer Engineering in 2010 and 2014. He is currently a Ph.D. Candidate in Electrical and Computer Engineering, Canada. Email:ajebelli@uottawa.ca

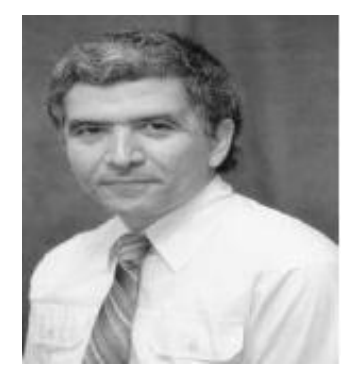

Mustapha C.E. Yagoub received the Dipl.-Ing. degree in Electronics and the Magister degree in Telecommunications, both from the École Nationale Polytechnique, Algiers, Algeria, in 1979 and 1987, respectively, and the Ph.D. degree from the Institut National Polytechnique, Toulouse, France, in 1994. After few years working in industry as a design engineer, he joined the Institute of Electronics, Université des Sciences et de la Technologie Houari Boumédiene, Algiers, Algeria, first as a Lecturer during 1983-1991 and then as an Assistant Professor during 1994-1999. From 1996 to 1999 , he has been head of the communication department. From 1999 to 2001, he was a visiting scholar with the Department of Electronics, Carleton University, Ottawa, ON, Canada, working on neural networks applications in microwave areas. In 2001, he joined the School of Information Technology and Engineering (SITE), University of Ottawa, Ottawa, ON, Canada, where he is currently a Professor. Dr. Yagoub is an Editorial Board member of the International Journal of RF and Microwave Computer-Aided Engineering, a senior member of the IEEE, and a registered member of the Professional Engineers of Ontario, Canada.

Email:myagoub@eecs.uottawa.ca

Dr. Dhillon has served as Chairman/Director/Acting Director of the Mechanical Engineering Department/Engineering Management Program for over 10 years. He is the founder of the probability distribution named Dhillon distribution by statistical researchers in their publications around the world. Dr. Dhillon has published over 380 \{i.e., 229 (70 single authored +159 co-authored) journal +151 conference proceedings $\}$ articles and 42 books: Wiley (1981), Van Nostrand (1983), Marcel Dekker (1984), Pergamon (1986), etc. His books are being used in over 100 countries and many of them are translated into languages such as German, Russian, Persian and Chinese. He is /has been on the editorial boards of 11 international scientific journals. Dr. Dhillon is recipient of many awards/honours including Austin J. Bonis Award (American Society for Quality Control), Merit Award (Society of Reliability Engineers), and Glinski Award (Faculty of Engineering). He is listed in many Who's Who documents and has served as consultant to various organizations. Professor Dhillon has lectured in over 50 countries including keynote addresses at various scientific conferences held in North America, Europe, Asia, and Africa. In March 2004, Dr. Dhillon was a distinguished speaker at the Conf./Workshop on Surgical Errors (sponsored by White House Health and Safety Committee and Pentagon), held at the Capitol Hill (One Constitution Avenue, Washington, D.C.).

Email: dhillon@genie.uottawa.ca 